\title{
The Grain of Vision and the Grain of Attention
}

\author{
Ned Block \\ New York University
}

Often when there is no attention to an object, there is no conscious perception of it either, leading some to conclude that conscious perception is an attentional phenomenon. There is a well-known perceptual phenomenon-visuo-spatial crowding, in which objects are too closely packed for attention to single out one of them. This article argues that there is a variant of crowding - what I call "identity-crowding" - in which one can consciously see a thing despite failure of attention to it. This conclusion, together with new evidence that attention to an object occurs in unconscious perception, suggests there may be a double dissociation between conscious perception of an object and attention to that object, constraining the extent to which consciousness can be constitutively attentional. The argument appeals to a comparison between the minimal resolution (or "grain") of object-attention and object-seeing.

DOI:10.1002/tht.28

\section{Introduction}

Dramatic phenomena such as "inattentional blindness" (Mack and Rock 1998) and the "attentional blink" (Raymond, Shapiro et al. 1992), in which stimuli that are not attended apparently elude conscious perception, have led many theorists to suppose that consciousness requires attention or even that conscious perception is constitutively attentional (Noë 2004; Tye 2010; Dehaene and Changeux 2011; O’Regan 2011; Cohen, Cavanagh et al. 2012; Prinz 2012).

This article will appeal to one variant of crowding (which I call "identity-crowding") to argue that there can be conscious perception of an object without attention to that object. It may seem obvious that we can see something without attending to it - for example, when one sees the minute-hand on a clock while attending to the second-hand. But it is hard to be sure whether one is attending to both or, if indeed one is not attending to the minute-hand, whether one is actually seeing it. I will sidestep such issues by appealing to a systematic phenomenon in which one sees something without even the possibility of attending to it.

\section{What is crowding?}

If you fixate one of the minuses on the left in Figure 1, you will be unable to identify or attend to the middle object in the group of three objects to the right. 


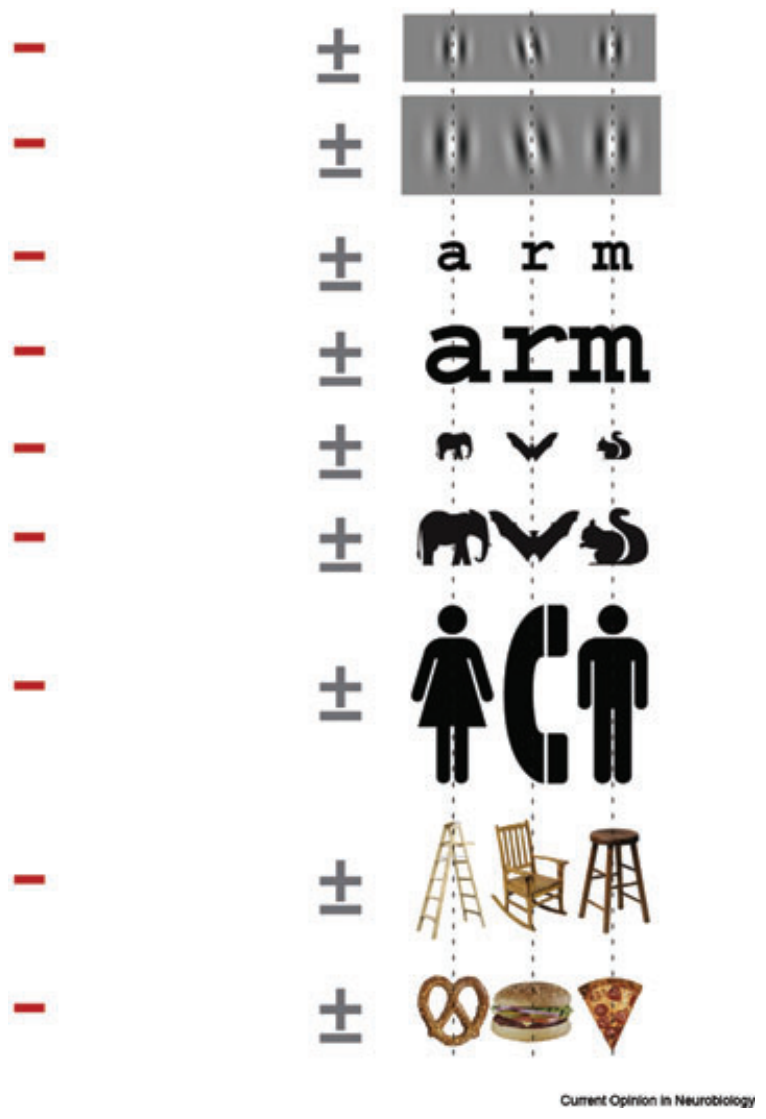

Figure 1: Fixating on (i.e., pointing your eyes at) one of the minuses on the left, you will be unable to identify the middle item in each row (and thus it is said to be "crowded") unless you isolate it by occluding the flanking objects with, for example, blank pieces of paper. Fixating on the \pm or perhaps slightly to the right side of it, you should be able to recognize the middle item. Note that the stimuli vary in size: at a given eccentricity (the angle from the eye between fixation and the center of the object), crowding is to a large extent independent of object size. Within limits, what counts for crowding is center-to-center distance measured by angle of vision from the eye. Crowding results from more than one object in a "window of integration" of features. Although one cannot identify the crowded objects in most cases of crowding, one can detect them in the sense that one can tell, visually, the difference between presence and absence. In this and other figures in this article, the reader is urged to hold the page at normal reading distance (about 14 inches from the eyes). Reprinted with permission from Pelli (2008): Elsevier.

Here is why: to see an individual in a crowded field, one's visual system must determine which features go with which objects. But there are minimal spatial areas - "windows of integration" - within which features can be identified but not assigned to more than one object (Intriligator and Cavanagh 2001; Pelli, Palomares et al. 2004; Levi 2008). Failure to correctly bind features to objects within a window results in objects looking "like a 
big mess" (Pelli, Palomares et al. 2004). Thus viewers often confuse the properties of flankers with properties of flanked items (Whitney and Levi 2011).

What do crowded stimuli look like? Things lose the quality of "form ... without losing crispness..." (Lettvin 1976). Subjects say things like "It looks like one big mess ... I seem to take features of one letter and mix them up with those of another." Or: "I know that there are three letters. But for some reason, I can't identify the middle one, which looks like it's being stretched and distorted by the outer flankers." (Pelli, Palomares et al. 2004, p. 1139).

In cases of crowding like those in Figure 1, the viewer cannot easily identify crowded items, but nonetheless the viewer can consciously detect crowded objects in the sense of consciously knowing the difference between presence and absence.

\section{The interaction between attention and crowding}

There are many typologies of attention, only one of which will matter here: object-based (in which attention selects an object), feature-based (in which attention selects a feature) and spatial (in which attention selects a spatial area). Object-based attention is what this article is mainly about, since I am arguing for a case in which there is object-seeing without object-(based) attention. It is attention that selects a single object and spreads to other parts of that object. (An illustration will be mentioned in the caption to Figure 9.) Feature-based attention can avoid crowding — for some features - as one can see in Figure 2. The ' $D$ ' on the left can be attended and hence consciously identified and discriminated from the other letters, while the ' $\mathrm{D}$ ' on the right cannot.

\section{Conscious versus unconscious}

On some views of crowding, perception of crowded items is often unconscious. Sid Kouider and his colleagues say that crowded items have "informational content in the absence of a corresponding conscious experience."(Faivre and Kouider 2011, p. 2) Their argument is based on failure of discrimination of crowded features from features of the flankers. However, discrimination can fail because conscious feature-representations of target and flankers are jumbled or merged. Speaking of a tilted grid flanked by and crowded by horizontal grids, Parkes, Lund et al. (2001) note that there is an experience of clockwise tilt ascribed to the group consisting of flankers and crowded item even though the crowded item does not itself seem visible: "Despite this invisibility, an overall impression of clockwise tilt may be seen.” (2001, p. 740) Kouider and his colleagues sometimes sound as if they agree, since they describe crowded features as "integrated into a subjectively jumbled texture" (2011, p. 7) and they regard crowding as "a typical situation of partial consciousness..." (2011, p. 8).

One may not be able to discriminate the crowded letter in row 4 of Figure 1 from other letters but one appreciates consciously and visually that the middle letter in row 4 is not face-like and that the middle item in the bottom row is not letter-like. The upshot is that at least in the cases discussed so far it is a mistake to regard the properties of the crowded items as merely unconsciously perceived. 


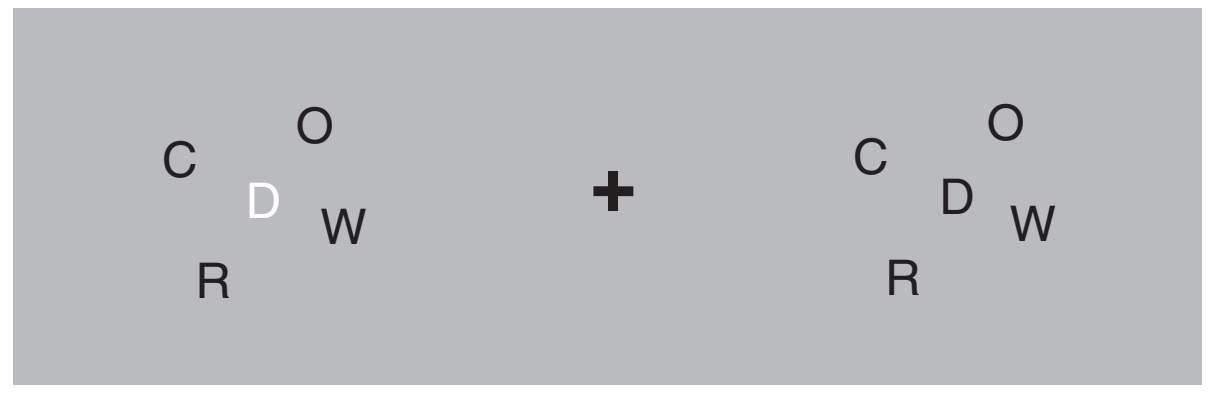

Figure 2: Fixating the cross at the center, one can make out the ' $D$ ' on the left much more easily than the ' $D$ ' on the right. Viewers can report the ' $D$ ' on the left because the difference in color allows feature-based attention to select it despite the fact that it is within the same window of integration as the surrounding letters. The fact that feature-based attention can select the ' $D$ ' on the left but spatial attention cannot select the ' $D$ ' on the right suggests that crowding involves a limit on spatial attention. Reprinted with permission from Whitney and Levi (2011): Elsevier.

\section{Textures}

Crowded items are often submerged into "textures" rather than being represented as objects. As Parkes et al. (2001) put it (p. 742), “... crowding and texture perception are opposite sides of the same coin." And this view of crowding is endorsed by Patrick Cavanagh in a companion article to Parkes aptly titled "Seeing the forest but not the trees" (Cavanagh 2001).

It may be said that we do not count as seeing an object if we see it as part of a texture. This would be a fatal objection because I am claiming that in one kind of crowding, one sees an individual object — as part of a pattern — but without attending to it. Consider the stars in a distant flag. You see all of them, collectively, but does that entail that you see any one of them, individually (Dretske 1969; Tye 2009a, 2009b)? Whether or not seeing a group requires seeing its individuals, the cases described in the next section are ones in which one sees both a group pattern and the individuals comprising it.

\section{Identity-crowding}

My case for object-seeing without object-attention depends on the kind of crowding illustrated in Figure 3.

Object-based attention cannot select the middle ' $\mathrm{A}$ ' in the bottom row (though there may be diffuse spatial attention to the area) but one can nonetheless identify the middle 'A'. Figure 4 illustrates another case of identity-crowding. You can't attend to a line in the middle of Figure 4 because your minimal window of integration - and spatial attention - is too large to select just one of the lines.

Patrick Cavanagh and his colleagues (2001) have operationally characterized the minimum grain of spatial attention. Their procedure is to ascertain whether the subject can "step" from one line to another. In "stepping", the subject is asked to maintain 


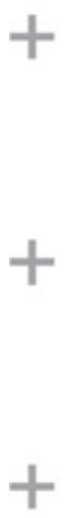

\section{XAX}

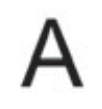

\section{AAA}

Figure 3: Fixate on each ' + '. You will find that you cannot identify the ' $A$ ' in the top row though it is recognizable in the bottom row and even easier to see in the middle row where it can be attentionally selected. The explanation for the effect of identity on crowding is straightforward. Crowding arises from failure of the visual system to determine which properties go with which objects, but when the items are identical, there is no such confusion. Identity-crowding, as one might call this, is not typical of crowding since there is no failure of identification. But since the inability to attend to the crowded items is, I believe, a more theoretically significant feature, I will describe identity-crowding as a form of crowding.

fixation on the cross (success can be checked with an eye-tracker). One line briefly changes color. Subjects are then asked to move their attention, one item at a time to the left or right. After a series of 5-7 randomly chosen moves of attention, one item changes color again and the subject is asked whether that is the one on which attention had alighted. The minimal spacing (in terms of angle from the eye) at which subjects can do this reliably is called the grain of attention.

We could define a corresponding notion of the grain of vision in terms of whether, in uncrowded fields, the subject can distinguish one dot from two dots, or whether the subject can detect a gap between two disks; or whether the subject can detect whether two dots that are close to one another and moving around a point in between them are rotating clockwise or counter-clockwise. These common methods of testing acuity yield different results that have something relevant in common: they are all smaller than the grain of attention. Further, the grain of attention degrades with eccentricity to a much larger extent than the grain of vision by any of these measures.

There is some disagreement as to whether the minimal windows of integration can be identified with the minimal grain of attention (Intriligator and Cavanagh 2001) or whether the minimal windows of integration are anatomically fixed (Pelli 2008).

The fact that identity-crowding allows identification is obvious from Figures 3-5. It was also shown experimentally using triples of grids each of which could be tilted either slightly clockwise or slightly counterclockwise (Petrov and Popple 2007). The triples were viewed in peripheral vision and subjects had to choose which of eight sequences of tilted grids was the one they saw in peripheral vision. (The eight were $\backslash \backslash 1, \backslash \backslash /, \backslash / /, \backslash / \backslash$ and their mirror images.) Since identification errors can be ones in which the middle item 

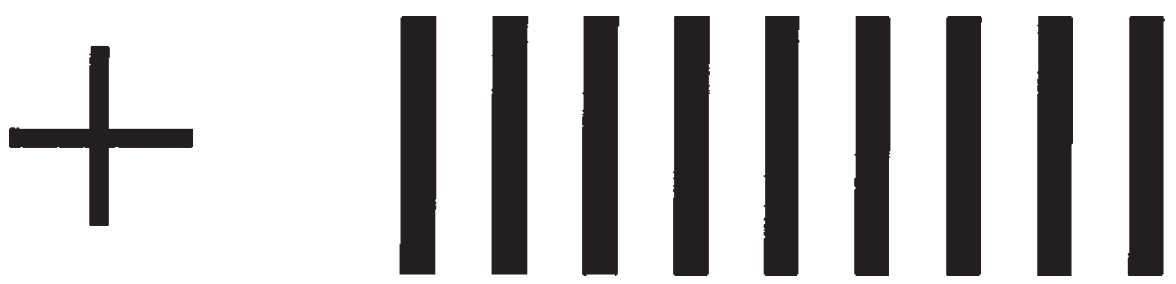

Figure 4: Fixate the cross and try to count the lines starting at the left. You can visually appreciate the approximate number but how many are there exactly? Try to attend to the leftmost bar and count the bars, moving your attention one by one to the right. You will find that you cannot do it so long as you continue fixating on the plus. It is easy if you move your eyes. There is a notable difference between this and earlier stimuli: each of the lines is a potential flanker and each of the lines that are not at the ends is a potential "target" of crowding. Whereas the grain of attention is three times coarser than the grain of vision at the fovea, at $15^{\circ}$ the grain of attention is 20 times coarser. That explains the "dramatic" (Intriligator and Cavanagh 2001, p. 203) impact of Figure 4. The middle of the group of lines is about $7^{\circ}$ from fixation, which makes the spacing of lines well above the grain of vision and well below the grain of attention. It is possible that acuity in crowded stimuli is different from acuity in uncrowded stimuli but this has not been tested as far as I know. I am assuming that objects that are more crowded than the grain of attention are not subject to object-based attention; a direct test of this assumption (one that has not been done as far as I know) would be to see if attention spreads from one part to another of a given line or other crowded item. This figure is also discussed in Block (2001). Reprinted with permission from Intriligator and Cavanagh (2001): John Wiley \& Sons, Inc.

affects identification of the flankers as well as when the flankers affect the middle item, the authors compared identifications of whole triples. One particularly dramatic result was that subjects were $96 \%$ correct if all the items slanted right $(/ / /)$, but only $53 \%$ correct if the middle item pointed in the opposite direction $(/ \backslash /)$. The fine structure of the results of this experiment suggests that subjects' success in identity crowding is genuinely perceptual and not just a cognitive inference from the look of uniformity and identification of the flankers. For there are considerable asymmetries between left and right tilts that only can be explained perceptually.

Since identity-crowding allows detection (i.e., distinguishing between presence and absence), differentiation from the background, discrimination from other items and visual identification of the items - all consciously - it is difficult to see a rationale for denying that one can consciously see them. (See Figure 5.)

It is uncontroversial that crowded items are below the grain of attention but it is controversial whether you can see the identity-crowded items. Patrick Cavanagh has argued that objects spaced more finely than the grain of attention "can only be perceived as a grouped texture" (Cavanagh, He et al. 1999, p. 41; Intriligator and Cavanagh 2001). Since he thinks one can attend to that texture and also consciously see it, he says there is no divergence between consciousness and attention. He appeals to the claim that the identity-crowded items cannot "be individuated for further processing" and that we cannot "access" or count or "inspect" the individual elements (Cavanagh, He et al. 1999, p. 41, 43). Counting certainly depends on attention but he gives no reason why counting 


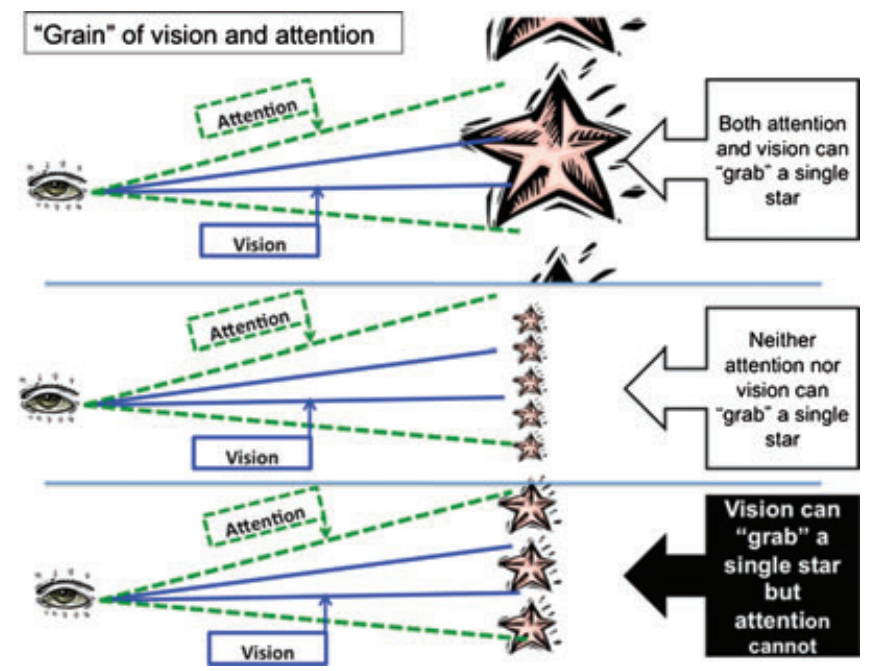

Figure 5: Think of the pairs of lines, dashed in the case of attention, solid in the case of vision, as the "tongs" of attention and vision. The openings depicted are the minimal grains of attention and vision. The tongs can be opened wider but, crucially, cannot be made narrower. In the top panel, both the tongs of vision and attention can "grab" a star, although the tongs of vision have to open up in order to do so. Thus those stars can be both seen and attended. In the middle panel, neither the tongs of vision nor attention can grab a single star. The stars are so small that there will always be more than one grabbed by the tongs. The bottom panel is the interesting one. The stars can be grabbed by the tongs of vision but not attention, that is, they can be seen but not attended. This point solves one version of the traditional "speckled hen" problem by denying C.I. Lewis' famous claim that ". . . there can never be 'positive bafflement' in the presence of the immediate, because there is here no question which fails to find an answer." (Lewis 1929, p. 128).

is required for object-seeing. I don't know what he means by "access", "inspect" or "individuate" if not something that depends on attending.

One can see the middle letter in the bottom row of Figure 3 well enough to be pretty sure it is an 'A' and the lines in Figure 4 well enough to know that, for example, they are not tilted at $45^{\circ}$ or horizontal. But does one see a letterish texture in the bottom of Figure 3 or on the left in Figure 6? Maybe a texture can be letterish but can it be A-ish or Times-Roman-A-ish? If one allows such detailed textures, it is not clear that there is any incompatibility between seeing textures and seeing objects that compose them. One can see an object and at the same time see it as fitting into a pattern that includes other objects.

\section{The concept of seeing an object}

To see something it must be visually differentiated from a background (Dretske 1969; Siegel 2006). Michael Tye (2010, p. 414) considers a version of Figure 4 (Cavanagh, He et al. 1999) noting that one cannot count the bars: "Why not? Surely because it is 


\section{A A \\ A $\mathrm{A}$}

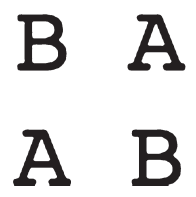

Figure 6: The A's on the left "escape crowding" because of identity: they are differentiated from the background, detectable, discriminable from other letters and identifiable. The letters on the both sides are crowded but without as much locational uncertainty as in Figure 4. Unlike the crowded items of Figure 4, Figure 3 and Figure 1, these items are not hemmed in from opposite directions. Reprinted with permission from Pelli et al. (2004): the Association for Research in Vision and Ophthalmology.

not the case that each bar on the right is clearly marked out or differentiated in the phenomenology of your experience. But then surely even though you are conscious of the bars on the right, it is not true that you are conscious of each individual bar." If the differentiation here is supposed to be differentiation between foreground and background, then not even two "surely"s can counteract the obvious fact that the lines of Figure 4 and the middle 'A' of Figure 3 are differentiated in one's experience from the background. One can see the white space in between the items. Tye also claims that one cannot see the crowded item because one cannot "mentally point" to it, but that just seems a way of saying that one cannot attend to it, and seeing without attention is just what is at issue.

It may be said that one does not visually locate any individual crowded line of Figure 4 well enough to distinguish it from all other lines. Maybe so, but this issue does not affect the middle 'A' in the bottom row of Figure 3 or the A's at the left side of Figure 6. These cases show that the variant of crowding we are talking about is compatible with localizability.

Susanna Siegel (2006) (see also Peacocke 1981) suggests that a condition on seeing an object is that the percept has the potential to be the basis of a thought, of that object, that it has such and such properties, a de re thought that singles out an object. (A similar condition has also suggested by Dretske (2007) and Tye (2009a, 2009b, 2010).) By contrast, the thought that the tallest spy, whoever he may be, is a spy is not de re. (As Siegel notes, forming a de re thought or indeed any thought requires mental machinery that is not required for seeing.) Some cases of identity crowding pass the test. I can ask of the middle letter in Figure 3 or the bottom left letter of Figure 6 "What is that?" I can attend to the rough spatial area of these letters but since they are crowded below the grain of attention, not to the letters themselves. So there is no incompatibility between identity crowding and being a potential basis of de re thought.

The upshot is that the demands of detection, differentiation, discrimination, identification, localization and de re belief potential do not derail the claim that in the identity-crowding cases of the last three figures one can consciously see an object without attending to it. This fact suggests that to the extent that identity crowded displays are seen as textures, the individual items can also be individually perceived. This conclusion is also backed up by an experimental phenomenon to which I now turn. 


\section{Crowding and memory}

Jeremy Freeman and Denis Pelli compared the memory capacities of crowded and uncrowded stimuli using a paradigm in which an item in a stimulus is cued after it has disappeared from the screen (Griffin and Nobre 2003; Lamme 2003) (See Figure 7.).

The result that is most directly relevant to the issue of perception of a crowded display as a kind of texture-perception is that there is virtually no difference in changedetection between the crowded and uncrowded performance when there is a cue. Note the similarity of the two bars on the right hand side of each of the four panels in Figure 8. The subjects are able to retain about 6 of the items after the stimulus has
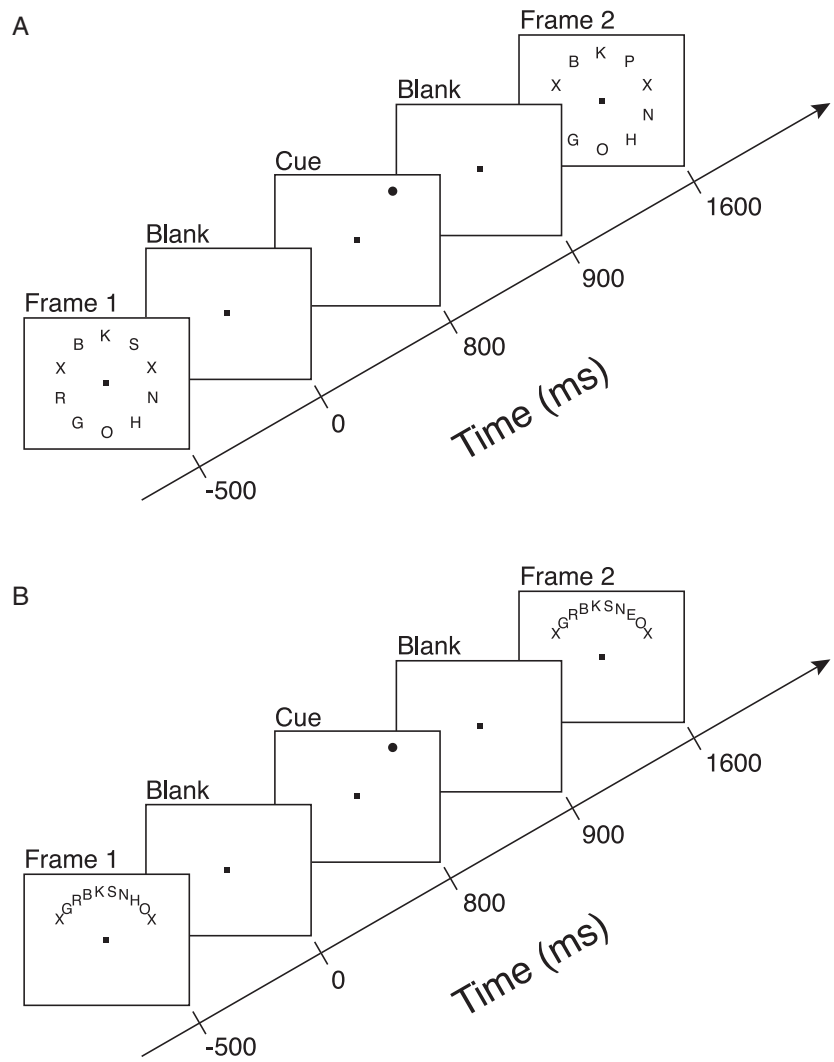

Figure 7: A display of letters is presented for $\mathbf{5 0 0}$ milliseconds, followed by an interval of $\mathbf{1 6 0 0}$ milliseconds, followed by a second display of letters, which in $50 \%$ of the trials, differs in one letter from the first display. The initial display is on the left. The subjects' task was to indicate whether any of the letters in the second display differed from the first display. In half the trials, there was a cue (a dot) 800 milliseconds after the offset of the initial frame that always indicated the location of the change when there was a change. Some of the displays used widely spaced letters (a) whereas others used crowded letters (b). Both (a) and (b) picture the cued case. Reprinted with permission from Freeman and Pelli (2007): the Association for Research in Vision and Ophthalmology. 

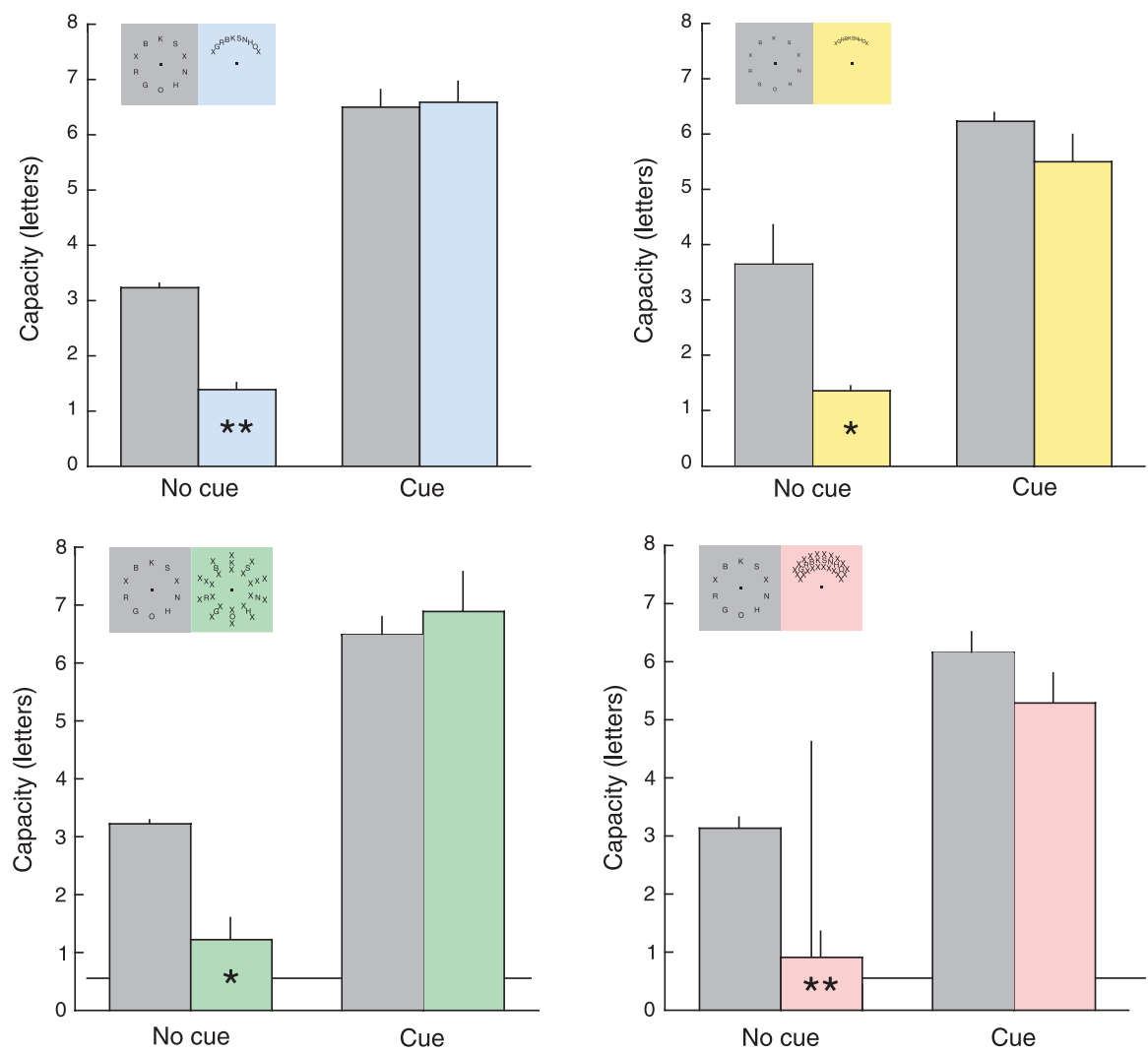

Figure 8: In each of these four panels, the gray column (on the left in each case) gives memory capacities for uncrowded stimuli and the colored columns (on the right) give memory capacities for various forms of crowded stimuli. The two striking results are (1) that there is little difference between crowded and uncrowded cued performance, and (2) that the crowded stimuli are much more strongly affected by the absence of a cue. The lack of an asterisk on the right hand side in each panel indicates that the difference between crowded and uncrowded cases does not reach statistical significance (conventionally set at a $5 \%$ probability of occurring by chance). There has been some controversy over whether this type of result depends illegitimately on practice effects (Matsukura and Hollingworth 2011) but both the crowded and uncrowded stimuli would have been subject to whatever practice effects are involved. Although subjects were excellent at detecting changes in both crowded and uncrowded stimuli, they were poor at reporting the uncrowded prechange letters and even worse at reporting the crowded prechange letters. $80 \%$ correct for changes, $40 \%$ identification for uncrowded letters, $25 \%$ identification for moderately crowded letters (blue, green and pink in Figure 8), and under 20\% identification for highly crowded letters (yellow in Figure 8). I have argued elsewhere (Block 2007, 2011) that conceptualization of a percept in working memory requires attention. My explanation - which I have no space to elaborate here-of these results would be that identification requires conceptualization, which requires attention; and crowding precludes attention. Reprinted with permission from Freeman and Pelli (2007): the Association for Research in Vision and Ophthalmology. 
disappeared whether or not they are crowded. It is hard to explain this result by appeal to texture-perception without letter-perception in the crowded case but not the uncrowded case. And denying letter perception in the uncrowded case is implausible and lacks independent motivation.

Freeman and Pelli did the experiment over again using unfamiliar (Armenian) letters, finding that the capacities were much lower and the difference between cued and uncued performance was greatly decreased. With English letters, cued capacities were about six, whereas with Armenian letters, they were reduced to a bit over two. Although the elements of Armenian letters are less familiar than those of English letters, there are many features in common, suggesting that the lack of difference between the crowded and uncrowded English letters depends on something in the direction of letter-recognition.

In this experiment, as in your own experience with crowded stimuli in Figure 1, conscious identification is poor. However, as noted earlier, one can be unable to distinguish a letter from other letters, but still be able to visually know the difference between letters and, say faces. Moreover, there are standard philosophical examples (Dretske 1969; Siegel 2006) such as seeing a far away object—say a house or a flock of geese--in poor light without being able to say much about what it is. So identification is not required for seeing.

Freeman and Pelli (2007p. 6) note "Observer S.B. reported that in both spacing conditions, the cue seemed to allow him to "bring back" the letter that had appeared in the cued location (take that with a grain of salt)." I concur on the grain of salt since S.B.'s comment does not reflect my own experience in this task. Despite this disagreement, there is one firm conclusion we can draw. Since unconscious seeing is still seeing, seeing an individual item is compatible with failure of object-based attention to it. So the Freeman and Pelli experiment shows that there can be seeing an object-even if unconscious seeing - without attention to that object.

If the cue promotes an unconscious representation into consciousness, presumably what is in consciousness before the cue would be a texture. But what is the unconscious representation before the cue? If it is not a letter (that is a letter-representation), perhaps it is an unorganized "bag" of isolated features (Oliva and Torralba 2007)? However, there is evidence against that hypothesis. First, features must be bound to a location in order to be locationally cued as in this experiment. Second, since many of the pairs of letters overlap a lot in features, the fact that performance does not depend on whether the letters are crowded or not suggests that the features are structured. But could there be an unorganized bag of unconscious features that is promoted to a structured conscious representation by attention? There is evidence (that I cannot summarize here) that representations of so many items could not persist in such a strong form for as long as 800 milliseconds (Block 2011).

Is "unconscious seeing" really seeing? And is this a substantial issue or merely verbal? My answers are: yes and substantial. In discussing seeing we should be focused on cutting nature at its joints. The best effort of this sort is Tyler Burge's (2009; 2010) characterization of seeing as a kind of objective sensory (in the visual modality) representation that is representation by the individual rather than by a subsystem. Seeing 


\begin{tabular}{|c|c|c|c|}
\hline Response & & Left & Difference on \\
\hline Same & 12 & 1 & 23 \\
\hline Different & 0 & 11 & 1 \\
\hline
\end{tabular}

Figure 9: "Visuo-spatial neglect" is a syndrome in which in many circumstances patients do not consciously see one side of space. Tony Ro showed a left-sided "neglect" patient various versions of the stimuli used in the Müller-Lyer illusion and the closely related Judd illusion. (In the Judd illusion, the brackets both face in the same direction, instead of in opposite directions as in the Müller-Lyer illusion). As you can see, the patient gave answers that with only a few exceptions did not involve the left sides of the figures. Ro asked this patient to bisect each of the lines in each of those figures. The different combinations of brackets have well confirmed effects on normal perceivers. (In the case of the Judd illusion, left-pointing brackets make the line appear to normal perceivers to protrude to the left, shifting the perceived center of the line to the left, and so lead to rightward bisection errors.) Interestingly, though the neglect patient showed the overall rightward drift on all figures characteristic of left sided neglect, he showed the normal pattern of influence by both the right and left brackets, showing that integrated conscious and unconscious perception of the brackets influenced the behavioral results. The syndrome of visuo-spatial neglect can be used to illustrate the basic nature of object based attention. If a subject like the one just described is shown a pair of disks, he will not see the disk on the left; but if a line is drawn connecting the disks, such subjects typically see the whole "dumbbell". Attention spreads from the right disk to the left disk since the visual system parses it as an object. Reprinted with permission from Ro and Rafal (1996): Elsevier.

so characterized does not require consciousness. Burge (2010) notes that we are sure that spiders see their prey and that bees see the famous waggle dance, but not sure the seeing is conscious. So we should conclude that our concept of seeing does not exclude unconscious seeing. Turning to humans and to the object-level rather than conceptual question, Burge notes that forms of unconscious perception involve some of the basic perceptual constancies, involving motion, location, and size.

One kind of dramatic evidence that conscious and unconscious seeing is of the same kind involves cases in which a single perceptual state involves integration of both conscious and unconscious elements. See Figures 9 and 10.

These points suggest that seeing both consciously and unconsciously belong to the same kind and that this is a substantive point. Thus there are no phenomenological requirements on object-seeing as Susanna Siegel and Michael Tye seem to suppose (Siegel 2006; Tye 2010). Further, the Freeman and Pelli experiment provides evidence against the claim that crowded seeing must be merely textural. To be clear: I have not argued against the possibility that what is in consciousness in Freeman and Pelli is merely textural until the cue promotes the letter-representation from unconscious to conscious perception. The role of Freeman and Pelli in my argument is to give an added boost to my case-made earlier - that in identity crowding, there is object-seeing without 

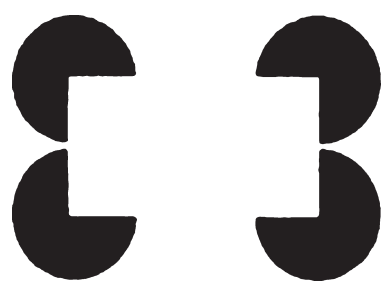

Figure 10: Kanizsa square. Normal perceivers see a rectangular surface when the four inducers are appropriately shaped and oriented. Vuillemier and Landis showed that neglect patients who could not consciously see the left-sided inducers could nonetheless see the rectangular surface, suggesting that conscious and unconscious perception can be integrated into a single percept. More complex results along the same lines can be seen in Conci, Böbel et al. (2009). Reprinted with permission from Vuilleumier and Landis (1998): NeuroReport.

object-attention. The boost derives from Freeman and Pelli's demonstration that objectseeing (if only unconscious object-seeing) is compatible with the lack of object-attention engendered by crowding. What would be damaging to my case is if there were evidence that in the Freeman and Pelli experiment, there was not only unconscious object-seeing before the cue but also unconscious object-attention at the same time. I know of no reason to believe this except to preserve the supposed attentional nature of perception.

To sum up, the identity-crowded items can be consciously detected, differentiated, discriminated and identified, while below the grain of attention, so those who deny that they are consciously seen owe us a better reason than that they are not object-attended. The only live alternative is texture-perception, but the Freeman and Pelli experiment suggests that crowded seeing does not have to be merely textural.

As I mentioned, object-based attention has been shown in some elegant experiments by Robert Kentridge and colleagues to operate in unconscious perception (Norman, Heywood et al. in press). My conclusion is that object-based attention may be doubly dissociated from consciously seeing an object. Given the elusiveness of broad spectrum conclusions about consciousness and attention, it may be that an approach stressing one or another kind of attention is the way to proceed.

\section{Acknowledgements}

I am grateful to the audience at Washington University, Chris Hill's class at the University of Pittsburgh, the Consciousness Project at NYU and a Harvard-MIT discussion group; and to the following persons for comments on an earlier draft: David Bennett, David Bitter, Eliza Block, Tyler Burge, Patrick Cavanagh, Eric Mandelbaum, Farid Masrour, John Morrison, Denis Pelli, Susanna Siegel and two anonymous reviewers.

\section{References}

Block, N. "Paradox and Cross Purposes in Recent Work on Consciousness." Cognition 79.1-2 (2001): 197-220. 
- "Consciousness, accessibility, and the mesh between psychology and neuroscience." Behavioral and Brain Sciences 30 (2007): 481-548.

—. "Perceptual Consciousness Overflows Cognitive Access." Trends in Cognitive Sciences 15.12 (2011): 567-75.

Burge, T. "Perceptual Objectivity.” Philosophical Review 118.3 (2009): 285-324.

. Origins of Objectivity. Oxford: Oxford University Press, 2010.

Cavanagh, P. "Seeing the Forest But Not the Trees.” Nature Neuroscience 4.7 (2001): 673-74.

Cavanagh, P., S. He, and J. Intriligator "Attentional Resolution: The Grain and Locus of Visual Awareness," in Neuronal Basis and Psychological Aspects of Consciousness, edited by C. Taddei-Ferretti and C. Musio. Singapore: World Scientific, 1999, 41-52.

Cohen, M., P. Cavanagh, M. Chun, and K. Nakayama. "The Attentional Requirements of Consciousness." Trends in Cognitive Sciences 16.8 (2012): 411-17.

Conci, M., E. Böbel, E. Matthias, I. Keller, H. Müller, and K. Finke. "Preattentive Surface and Contour Grouping in Kanizsa Figures: Evidence from Parietal Extinction.” Neuropsychologia 47 (2009): $726-32$.

Dehaene, S., and J. Changeux. "Experimental and Theoretical Approaches to Conscious Processing." Neuron 70 (2011): 200-27.

Dretske, F. Seeing and Knowing. Chicago: University of Chicago Press, 1969.

Dretske, F. "What Change Blindness Teaches about Consciousness?" Philosophical Perspectives 21 (2007): 215-30.

Faivre, N., and S. Kouider. "Multi-feature Objects Elicit Non-conscious Priming Despite Crowding." Journal of Vision 11.3 (2011): 1-10.

Freeman, J., and D. Pelli. “An Escape from Crowding.” Journal of Vision 7.2 (2007): 1-14.

Griffin, I. C., and A. C. Nobre. "Orienting Attention to Locations in Internal Representations." Journal of Cognitive Neuroscience 15 (2003): 1176-94.

Intriligator, J., and P. Cavanagh. "The Spatial Resolution of Visual Attention." Cognitive Psychology 43 (2001): 171-216.

Lamme, V. "Why Visual Attention and Awareness Are Different?" Trends in Cognitive Sciences 7 (2003): $12-18$.

Lettvin, J. Y. “On Seeing Sidelong." The Sciences 16.4 (1976): 10-20.

Levi, D. "Crowding — An Essential Bottleneck for Object Recognition: A Mini-Review.” Vision Research 48 (2008): 635-54.

Lewis, C. I. Mind and the World-Order: An Outline of a Theory of Knowledge. New York: Scribner's, 1929.

Mack, A., and I. Rock. Inattentional Blindness. Cambridge, MA: MIT Press, 1998.

Matsukura, M., and A. Hollingworth. "Does Visual Short-term Memory Have a High Capacity Stage?” Psychonomic Bulletin \& Review 18.6 (2011): 1098-104.

Noë, A. Action in Perception. Cambridge, MA: MIT Press, 2004.

Norman, L. J., C. Heywood., and R. Kentridge. “Object-Based Attention Without Awareness.” Psychological Science (in press).

O'Regan, J. K. Why Red Doesn't Sound Like a Bell: Understanding the Feel of Consciousness. New York: Oxford University Press, 2011.

Oliva, A., and A. Torralba. "The Role of Context in Object Recognition." Trends in Cognitive Sciences 11.12 (2007): 520-27.

Parkes, L., J. Lund, A. Angelucci, J. Solomon, and M. Morgan. "Compulsory Averaging of Crowded Orientation Signals in Human Vision.” Nature Neuroscience 4.7 (2001): 739-44. 
Peacocke, C. "Demonstrative Thought and Psychological Explanation." Synthese 49.2 (1981): 187-215.

Pelli, D. "Crowding: A Cortical Constraint on Object Recognition." Current Opinion in Neurobiology 18.4 (2008): 445-51.

Pelli, D., M. Palomares, and N. Majaj. "Crowding Is Unlike Ordinary Masking: Distinguishing Feature Integration from Detection.” Journal of Vision 4 (2004): 1136-69.

Petrov, Y., and A. V. Popple. "Crowding is Directed to the Fovea and Preserves Only Feature Contrast." Journal of Vision 7.2 (2007): 8.1-9.

Prinz, J. J. The Conscious Brain. New York: Oxford University Press, 2012.

Raymond, J., K. Shapiro., and K. Arnell. "Temporary Suppression of Visual Processing in an RSVP Task: An Attentional Blink?” Journal of Experimental Psychology: Human Perception and Performance 18.3 (1992): 849-60.

Ro, T., and R. Rafal. "Perception of Geometric Illusions in Hemispatial Neglect." Neuropsychologia 34.10 (1996): 973-78.

Siegel, S. "How Does Visual Phenomenology Constrain Object-Seeing?" Australasian Journal of Philosophy 84.3 (2006): 429-41.

Tye, M. Consciousness Revisited. Cambridge: MIT Press, 2009a.

_. "A New Look at the Speckled Hen." Analysis 69 (2009b): 258-63.

"Attention, Seeing and Change Blindness." Philosophical Issues 20.1 (2010): 410-37.

Vuilleumier, P., and T. Landis. "Illusory Contours and Spatial Neglect." Neuroreport 9.11 (1998): 2481-84.

Whitney, D., and D. Levi. "Visual Crowding: A Fundamental Limit on Conscious Perception and Object Recognition.” Trends in Cognitive Sciences 15.4 (2011): 160-68. 